\title{
Dynamics of blow-up solutions for the Schrödinger-Choquard equation
}

\section{Cunqin Shi* and Kun Liu}

\section{"Correspondence:} shicq1978@163.com

School of Mathematics and Statistic, Longdong University, Qingyang, P.R. China

\section{Springer}

\begin{abstract}
In this paper, we study the dynamics of blow-up solutions for the nonlinear Schrödinger-Choquard equation

$$
i \psi_{t}+\Delta \psi=\lambda_{1}|\psi|^{p_{1}} \psi+\lambda_{2}\left(l_{\alpha} *|\psi|^{p_{2}}\right)|\psi|^{p_{2}-2} \psi
$$

We first show existence of blow-up solutions and obtain a sharp threshold mass of global existence and blow-up for this equation with $\lambda_{1}>0, \lambda_{2}<0,0<p_{1}<\frac{4}{N}$ and $p_{2}=1+\frac{2+\alpha}{N}$. Then we obtain some dynamical properties of blow-up solutions by the corresponding ground state of this equation with $\lambda_{1}=0$.
\end{abstract}

MSC: 35Q55; 35A15

Keywords: Nonlinear Schrödinger-Choquard equation; Blow-up solutions; The dynamical properties

\section{Introduction}

In this paper, we will investigate the blow-up solutions of the nonlinear SchrödingerChoquard equation

$$
\left\{\begin{array}{l}
i \psi_{t}+\Delta \psi=\lambda_{1}|\psi|^{p_{1}} \psi+\lambda_{2}\left(I_{\alpha} *|\psi|^{p_{2}}\right)|\psi|^{p_{2}-2} \psi, \\
\psi(0, x)=\psi_{0}(x)
\end{array}\right.
$$

where $\psi(t, x):\left[0, T^{*}\right) \times \mathbb{R}^{N} \rightarrow \mathbb{C}$ is a complex valued function and $0<T^{*} \leq \infty, N \geq 3$, $\psi_{0} \in H^{1}, 0<p_{1}<\frac{4}{N-2}, 1+\frac{\alpha}{N}<p_{2}<1+\frac{2+\alpha}{N-2}, \lambda_{1}, \lambda_{2} \in \mathbb{R}, I_{\alpha}: \mathbb{R}^{N} \rightarrow \mathbb{R}$ is the Riesz potential defined by

$$
I_{\alpha}(x)=\frac{\Gamma\left(\frac{N-\alpha}{2}\right)}{\Gamma\left(\frac{\alpha}{2}\right) \pi^{N / 2} 2^{\alpha}|x|^{N-\alpha}},
$$

with $\max \{0, N-4\}<\alpha<N$ and $\Gamma$ is the Gamma function.

Our main motivation for studying Eq. (1.1) is the loss of scaling invariance for this equation. When $p_{2}>0$, there exists a scaling transform for the nonlinear Choquard equation,

$$
i \psi_{t}+\Delta \psi=\lambda_{2}\left(I_{\alpha} *|\psi|^{p_{2}}\right)|\psi|^{p_{2}-2} \psi
$$

(c) The Author(s) 2018. This article is distributed under the terms of the Creative Commons Attribution 4.0 International License (http://creativecommons.org/licenses/by/4.0/), which permits unrestricted use, distribution, and reproduction in any medium, provided you give appropriate credit to the original author(s) and the source, provide a link to the Creative Commons license, and indicate if changes were made. 
which keeps it invariant. More precisely, the map

$$
\psi(t, x) \mapsto \lambda^{-\frac{\alpha+2}{2 p_{2}-2}} \psi\left(\frac{t}{\lambda^{2}}, \frac{x}{\lambda}\right)
$$

maps a solution to (1.2) to another solution to (1.2). When $p_{2}=1+\frac{2+\alpha}{N}$, the scaling transform (1.3) keeps the mass invariant. Thus, the nonlinearity $\left(I_{\alpha} *|\psi|^{p_{2}}\right)|\psi|^{p_{2}-2} \psi$ is called $L^{2}$-critical.

When $\lambda_{1}=0$ and $p_{2}=2$, Eq. (1.1) simplifies to the Hartree equation. The Cauchy problem of (1.1) has been extensively investigated in [1-16]. The local well-posedness and global existence of (1.1) have been studied in [1]. Chen and Guo [3] studied the instability of standing waves. In the $L^{2}$-critical case, Miao et al. [10] studied the dynamical properties of the blow-up solutions. The soliton dynamics has been studied in [11].

When $\lambda_{1}=0,0<\alpha<N$ and $1+\frac{\alpha}{N}<p_{2}<\frac{N+\alpha}{N-2}$, under the assumption that the local wellposedness holds for (1.1), Chen and Guo [3] derived the existence of blow-up solutions and the instability of standing waves. When $0<\alpha<N$ and $1+\frac{\alpha}{N}<p_{2}<1+\frac{2+\alpha}{N}$, Squassina et al. in [17] studied the soliton dynamics of (1.1) under the assumption that the solution $\psi$ of (1.1) is in $C\left([0, \infty), H^{2}\right) \cap C^{1}\left((0, \infty), L^{2}\right)$. In [18], Feng and Yuan systematically studied the Cauchy problem (1.1) for general $\max \{0, N-4\}<\alpha<N$ and $2 \leq p_{2}<\frac{N+\alpha}{N-2}$. More precisely, they studied the local well-posedness, global existence, the existence of blow-up solutions and the dynamics of blow-up solutions. The sharp threshold of global existence and blowup, the instability of standing wave of (1.1) with $\lambda_{1}=0$ and a harmonic potential have been investigated in [19].

However, in the above papers, the scale invariance plays an important role in the study of the dynamics of blow-up solutions to (1.2); see [7, 10, 12, 14, 18, 20,21]. Because there exists no scale invariance for (1.1), the study of blow-up solutions to (1.1) is a very interesting problem. On the other hand, as far as we know, the existence of blow-up solutions to (1.1) with $\lambda_{1}>0, \lambda_{2}<0,0<p_{1}<\frac{4}{N}$ and $p_{2}=1+\frac{2+\alpha}{N}$ has not been obtained yet. Hence, in this paper, we first show the existence of blow-up solutions and obtain the sharp threshold mass $\|u\|_{L^{2}}$ of global existence and blow-up for (1.1), where $u$ is a ground state solution of the elliptic equation

$$
-\Delta u+u-\left(I_{\alpha} *|u|^{p}\right)|u|^{p-2} u=0 .
$$

Then, for overcoming the difficulty of the loss of scale invariance, we apply the ground state solution $u$ of (1.4) to describe the dynamical properties of blow-up solutions to (1.1), including $L^{2}$-concentration, limiting profile and blow-up rates.

This paper is organized as follows: in Sect. 2, we recall some preliminaries. In Sect. 3, we firstly show the existence of blow-up solutions to (1.1) with $\lambda_{1}=1, \lambda_{2}=-1,0<p_{1}<\frac{4}{N}$ and $p_{2}=1+\frac{2+\alpha}{N}$, and then obtain the sharp threshold mass $\|u\|_{L^{2}}$ of global existence and blow-up. In Sect. 4 , we will consider some dynamical properties of blow-up solutions to (1.1) with $\lambda_{1}=1, \lambda_{2}=-1,0<p_{1}<\frac{4}{N}$ and $p_{2}=1+\frac{2+\alpha}{N}$. Section 5 is a concluding section.

Notation In this paper, we use the following notations. We always denote $u$ the ground state solution of (1.4). $\Sigma:=\left\{\psi \in H^{1}, x \psi \in L^{2}\right\}$ is the energy space equipped with the norm $\|\psi\|_{\Sigma}:=\|\psi\|_{H^{1}}+\|x \psi\|_{L^{2}}$. 


\section{Preliminaries}

In order to study the blow-up solutions to (1.1), we firstly make the following assumption about the local well-posedness of (1.1).

Assumption 1 Let $\psi_{0} \in H^{1}, N \geq 3,0<p_{1}<\frac{4}{N-2}$ and $1+\frac{\alpha}{N}<p_{2}<1+\frac{2+\alpha}{N-2}$. Then there exist $T^{*}>0$ and a unique maximal solution $\psi \in C\left(\left[0, T^{*}\right), H^{1}\right)$. In addition, if $T^{*}<\infty$, then $\|\psi(t)\|_{H^{1}} \rightarrow \infty$ as $t \uparrow T^{*}$. Moreover, the solution $\psi(t)$ satisfies

$$
\begin{aligned}
& \|\psi(t)\|_{L^{2}}=\left\|\psi_{0}\right\|_{L^{2}}, \\
& E(\psi(t))=E\left(\psi_{0}\right),
\end{aligned}
$$

for all $0 \leq t<T^{*}$, where $E(\psi(t))$ is defined by

$$
\begin{aligned}
E(\psi(t)):= & \frac{1}{2} \int_{\mathbb{R}^{N}}|\nabla \psi(t, x)|^{2} d x+\frac{\lambda_{1}}{p_{1}+2} \int_{\mathbb{R}^{N}}|\psi(t, x)|^{p_{1}+2} d x \\
& +\frac{\lambda_{2}}{2 p_{2}} \int_{\mathbb{R}^{N}}\left(I_{\alpha} *|\psi|^{p_{2}}\right)(t, x)|\psi(t, x)|^{p_{2}} d x .
\end{aligned}
$$

When $0<p_{1}<\frac{4}{N-2}$ and $2 \leq p_{2}<1+\frac{2+\alpha}{N-2}$, this assumption can easily be proved by the Strichartz estimates and a fixed point argument; see $[1,18]$.

By the same argument as that in [1], one can easily derive the following lemma.

Lemma 2.1 ([1]) Let $\psi_{0} \in \Sigma:=\left\{u \in H^{1}, x u \in L^{2}\right\}$. Assume that the solution $\psi(t)$ to (1.1) exists on the interval $\left[0, T^{*}\right)$. Then $\psi(t) \in \Sigma$ for all $t \in\left[0, T^{*}\right)$. Moreover, let $J(t)=$ $\int_{\mathbb{R}^{N}}|x \psi(t, x)|^{2} d x$, then

$$
J^{\prime}(t)=-4 \operatorname{Im} \int_{\mathbb{R}^{N}} \psi(t, x) x \cdot \nabla \bar{\psi}(t, x) d x
$$

and

$$
\begin{aligned}
J^{\prime \prime}(t)= & 8 \int_{\mathbb{R}^{N}}|\nabla \psi(t, x)|^{2} d x+\frac{4 N \lambda_{1} p_{1}}{p_{1}+2} \int_{\mathbb{R}^{N}}|\psi(t, x)|^{p_{1}+2} d x \\
& +\lambda_{2} \frac{4 p_{2} N-4 N-4 \alpha}{p_{2}} \int_{\mathbb{R}^{N}}\left(I_{\alpha} *|\psi|^{p_{2}}\right)(t, x)|\psi(t, x)|^{p_{2}} d x
\end{aligned}
$$

As a direct result of this lemma, we have the following lemma.

Lemma 2.2 If the solution $\psi(t)$ to (1.1) with $\psi_{0} \in \Sigma$ blows up at the finite time $T^{*}$, then there exists $C>0$ such that for all $t \in\left[0, T^{*}\right)$

$$
\int_{\mathbb{R}^{N}}|x|^{2}|\psi(t, x)|^{2} d x \leq C
$$

Next, we summarize some results about the ground state of (1.4), which is very important in the study of blow-up solutions to (1.1). 
Lemma $2.3([17,22])$ Let $\alpha \in(0, N)$ and $1+\frac{\alpha}{N}<p<1+\frac{2+\alpha}{N-2}$. Then (1.4) admits a ground state solution $u$ in $H^{1}$. Moreover, let $u_{1}$ and $u_{2}$ be two any ground state solutions of (1.4), then $\left\|u_{1}\right\|_{L^{2}}=\left\|u_{2}\right\|_{L^{2}}$.

Finally, we recall a useful result which gives the best constant in a Gagliardo-Nirenberg type inequality; see [18].

Lemma 2.4 The best constant in the Gagliardo-Nirenberg type inequality

$$
\int_{\mathbb{R}^{N}}\left(I_{\alpha} *|\psi|^{p}\right)|\psi|^{p} d x \leq C_{\alpha, p}\left(\int_{\mathbb{R}^{N}}|\nabla \psi|^{2} d x\right)^{\frac{N p-N-\alpha}{2}}\left(\int_{\mathbb{R}^{N}}|\psi|^{2} d x\right)^{\frac{N+\alpha-N p+2 p}{2}}
$$

is

$$
C_{\alpha, p}=\frac{2 p}{2 p-N p+N+\alpha}\left(\frac{2 p-N p+N+\alpha}{N p-N-\alpha}\right)^{\frac{N p-N-\alpha}{2}}\|u\|_{L^{2}}^{2-2 p}
$$

In particular, in the $L^{2}$-critical case, i.e., $p=1+\frac{2+\alpha}{N}, C_{\alpha, p}=p\|u\|_{L^{2}}^{2-2 p}$.

\section{The sharp threshold mass of global existence and blow-up}

From the local well-posedness of the nonlinear Schrödinger-Choquard equation, for small initial data $\psi_{0}$, the solution $\psi(t)$ to (1.1) exists globally, and the solution $\psi(t)$ may blow up for some large initial data. Therefore, whether there are some sharp thresholds of global existence and blow-up for (1.1) is a very interesting problem. In particular, the sharp thresholds of global existence and blow-up for nonlinear Schrödinger equations are pursued strongly (see [1, 2, 19, 23-25] and the references therein).

In the following, applying the inequality (2.6) and a scaling argument, we derive the existence of blow-up solutions to (1.1) and a sharp threshold of global existence and blowup.

Theorem 3.1 Let $\psi_{0} \in H^{1}, \lambda_{1}=1, \lambda_{2}=-1,0<p_{1}<\frac{4}{N}$ and $p_{2}=1+\frac{2+\alpha}{N}$. Then we have:

(i) If $\left\|\psi_{0}\right\|_{L^{2}}<\|u\|_{L^{2}}$, then the solution $\psi(t)$ to (1.1) exists globally.

(ii) Let $\psi_{0}=c \rho^{\frac{N}{2}} u(\rho x)$ and $|x| \psi_{0} \in L^{2}$, where $|c| \geq 1$, and $\rho>0$ and satisfies

$$
\frac{2|c|^{p_{1}}\|u\|_{L^{p_{1}+2}}^{p_{1}+2}}{\left(p_{1}+2\right)\left(|c|^{2 p_{2}-2}-1\right)\|\nabla u\|_{L^{2}}^{2}}<\rho^{2-\frac{N}{2} p_{1}}
$$

Then the solution $\psi(t)$ to (1.1) blows up in finite time.

Remark We see from Theorem 1.2 in [18] that the critical value about the initial data for global existence of (1.1) with $\lambda_{1}=0$ and (1.1) is the same.

Proof (i) Firstly, by (2.3) and (2.6), we have

$$
\begin{aligned}
E\left(\psi_{0}\right) & =E(\psi(t)) \\
& =\frac{1}{2} \int_{\mathbb{R}^{N}}|\nabla \psi(t, x)|^{2} d x-\frac{1}{2 p_{2}} \int_{\mathbb{R}^{N}}\left(I_{\alpha} *|\psi|^{p_{2}}\right)(t, x)|\psi(t, x)|^{p_{2}} d x
\end{aligned}
$$




$$
\begin{gathered}
+\frac{1}{p_{1}+2} \int_{\mathbb{R}^{N}}|\psi(t, x)|^{p_{1}+2} d x \\
\geq\left(\frac{1}{2}-\frac{\left\|\psi_{0}\right\|_{L^{2}}^{2 p_{2}-2}}{2\|u\|_{L^{2}}^{2 p_{2}-2}}\right)\|\nabla \psi(t)\|_{L^{2}}^{2} .
\end{gathered}
$$

It follows from $\left\|\psi_{0}\right\|_{L^{2}}<\|u\|_{L^{2}}$ and $E\left(\psi_{0}\right)=E(\psi(t))$ that there exists a constant $C$ such that $\|\nabla \psi(t)\|_{L^{2}} \leq C$ for all $t>0$. Therefore, the solution $\psi(t)$ to (1.1) exists globally.

(ii) Since $|x| \psi_{0} \in L^{2}, J(t)=\int_{\mathbb{R}^{N}}|x \psi(t, x)|^{2} d x$ is well defined. We deduce from Lemma 2.1 that

$$
J^{\prime \prime}(t)=16 E\left(\psi_{0}\right)-\frac{16-4 N p_{1}}{p_{1}+2} \int_{\mathbb{R}^{N}}|\psi(t, x)|^{p_{1}+2} d x .
$$

Since $\psi_{0}(x)=c \rho^{\frac{N}{2}} u(\rho x)$ and the Pohožaev identity of (1.4), i.e., $\frac{1}{2} \int_{\mathbb{R}^{N}}|\nabla u(x)|^{2} d x=$ $\frac{1}{2 p_{2}} \int_{\mathbb{R}^{N}}\left(I_{\alpha} *|u|^{p_{2}}\right)(x)|u(x)|^{p_{2}} d x$ (see [18]), it follows that

$$
\begin{aligned}
E\left(\psi_{0}\right)= & \frac{|c|^{2} \rho^{2}}{2} \int_{\mathbb{R}^{N}}|\nabla u(x)|^{2} d x-\frac{|c|^{2 p_{2}} \rho^{2}}{2 p_{2}} \int_{\mathbb{R}^{N}}\left(I_{\alpha} *|u|^{p_{2}}\right)(x)|u(x)|^{p_{2}} d x \\
& +\frac{|c|^{p_{1}+2} \rho^{\frac{N}{2} p_{1}}}{p_{1}+2} \int_{\mathbb{R}^{N}}|u(x)|^{p_{1}+2} d x \\
= & -\frac{|c|^{2} \rho^{2}}{2}\left(|c|^{2 p_{2}-2}-1\right)\|\nabla u\|_{L^{2}}^{2}+\frac{|c|^{p_{1}+2} \rho^{\frac{N}{2} p_{1}}}{p_{1}+2} \int_{\mathbb{R}^{N}}|u(x)|^{p_{1}+2} d x .
\end{aligned}
$$

Thus, it follows from (3.1) that $E\left(\psi_{0}\right)<0$. We deduce from (3.2) that $J^{\prime \prime}(t)<16 E\left(\psi_{0}\right)<0$. By a standard argument, the solution $\psi(t)$ to (1.1) with $\psi_{0}=c \rho^{\frac{N}{2}} u(\rho x)$ blows up in finite time.

\section{Dynamics of blow-up solutions in the $L^{2}$-critical case}

In this section, we study the dynamical properties of blow-up solutions for (1.1) with $\lambda_{1}=1, \lambda_{2}=-1,0<p_{1}<\frac{4}{N}$ and $p_{2}=1+\frac{2+\alpha}{N}$. For this purpose, we firstly recall a refined compactness lemma which has been proved in [18] by the inequality (2.6) and the profile decomposition theory.

Lemma 4.1 Let $p_{2}=1+\frac{2+\alpha}{N}$. If $\left\{\psi_{n}\right\}_{n=1}^{\infty}$ is a bounded sequence in $H^{1}$ and satisfies

$$
\limsup _{n \rightarrow \infty}\left\|\nabla \psi_{n}\right\|_{L^{2}}^{2} \leq M, \quad \limsup _{n \rightarrow \infty} \int_{\mathbb{R}^{N}}\left(I_{\alpha} *\left|\psi_{n}\right|^{p_{2}}\right)\left|u_{n}\right|^{p_{2}} d x \geq m
$$

Then there exists $\left\{x_{n}\right\}_{n=1}^{\infty} \subset \mathbb{R}^{N}$, such that, up to a subsequence,

$$
\psi_{n}\left(\cdot+x_{n}\right) \rightarrow \Psi
$$

with $\|\Psi\|_{L^{2}} \geq\left(\frac{m}{p_{2} M}\right)^{\frac{1}{2 p_{2}-2}}\|u\|_{L^{2}}$.

Theorem 4.2 ( $L^{2}$-concentration) Assume that $\psi_{0} \in H^{1}, \lambda_{1}=1, \lambda_{2}=-1,0<p_{1}<\frac{4}{N}$ and $p_{2}=1+\frac{2+\alpha}{N}$. Let the solution $\psi(t)$ to (1.1) blow up at the finite time $T^{*}$. If $a(t):\left[0, T^{*}\right) \mapsto \mathbb{R}$ 
is a real-valued function and $a(t)\|\nabla \psi(t)\|_{L^{2}} \rightarrow \infty$ as $t \rightarrow T^{*}$. Then there exists $x(t) \in \mathbb{R}^{N}$ such that

$$
\liminf _{t \rightarrow T^{*}} \int_{|x-x(t)| \leq a(t)}|\psi(t, x)|^{2} d x \geq \int_{\mathbb{R}^{N}}|u(x)|^{2} d x
$$

Proof Set

$$
\rho_{n}:=\|\nabla u\|_{L^{2}} /\left\|\nabla \psi\left(t_{n}\right)\right\|_{L^{2}} \quad \text { and } \quad v_{n}(x):=\rho_{n}^{\frac{N}{2}} \psi\left(t_{n}, \rho_{n} x\right)
$$

where $\left\{t_{n}\right\}_{n=1}^{\infty} \subseteq\left[0, T^{*}\right)$ and $t_{n} \rightarrow T^{*}$ as $n \rightarrow \infty$. Then the sequence $\left\{v_{n}\right\}$ satisfies

$$
\begin{aligned}
& \left\|v_{n}\right\|_{L^{2}}=\left\|\psi\left(t_{n}\right)\right\|_{L^{2}}=\left\|\psi_{0}\right\|_{L^{2}}, \\
& \left\|\nabla v_{n}\right\|_{L^{2}}=\rho_{n}\left\|\nabla \psi\left(t_{n}\right)\right\|_{L^{2}}=\|\nabla u\|_{L^{2}} .
\end{aligned}
$$

It follows from (2.3) that

$$
\begin{aligned}
H\left(v_{n}\right) & :=\frac{1}{2} \int_{\mathbb{R}^{N}}\left|\nabla v_{n}(x)\right|^{2} d x-\frac{1}{2 p_{2}} \int_{\mathbb{R}^{N}}\left(I_{\alpha} *\left|v_{n}\right|^{p_{2}}\right)(x)\left|v_{n}(x)\right|^{p_{2}} d x \\
& =\rho_{n}^{2}\left(\frac{1}{2} \int_{\mathbb{R}^{N}}\left|\nabla \psi\left(t_{n}, x\right)\right|^{2} d x-\frac{1}{2 p_{2}} \int_{\mathbb{R}^{N}}\left(I_{\alpha} *\left|\psi\left(t_{n}\right)\right|^{p_{2}}\right)(x)\left|\psi\left(t_{n}, x\right)\right|^{p_{2}} d x\right) \\
& =\rho_{n}^{2}\left(E\left(\psi_{0}\right)-\frac{1}{p_{1}+2} \int_{\mathbb{R}^{N}}\left|\psi\left(t_{n}, x\right)\right|^{p_{1}+2} d x\right) .
\end{aligned}
$$

Hence, by the Gagliardo-Nirenberg inequality

$$
\int_{\mathbb{R}^{N}}|\psi(x)|^{p_{1}+2} d x \leq C\|\psi\|_{L^{2}}^{p_{1}+2-\frac{N p_{1}}{2}}\|\nabla \psi\|_{L^{2}}^{\frac{N p_{1}}{2}}
$$

and $0<p_{1}<\frac{4}{N}$, it follows that

$$
\begin{aligned}
\left|H\left(v_{n}\right)\right| & \leq \rho_{n}^{2}\left(\left|E\left(\psi_{0}\right)\right|+\frac{1}{p_{1}+2} \int_{\mathbb{R}^{N}}\left|\psi\left(t_{n}, x\right)\right|^{p_{1}+2} d x\right) \\
& \leq \frac{\left|E\left(\psi_{0}\right)\right|\|\nabla u\|_{L^{2}}^{2}}{\left\|\nabla \psi\left(t_{n}\right)\right\|_{L^{2}}^{2}}+C \frac{\|\nabla \psi\|_{L^{2}}^{2}\left\|\nabla \psi\left(t_{n}\right)\right\|_{L^{2}}^{\frac{N p_{1}}{2}}}{\left\|\nabla \psi\left(t_{n}\right)\right\|_{L^{2}}^{2}} \rightarrow 0 \quad \text { as } n \rightarrow \infty .
\end{aligned}
$$

This yields $\int_{\mathbb{R}^{N}}\left(I_{\alpha} *\left|v_{n}\right|^{p_{2}}\right)\left|v_{n}\right|^{p_{2}} d x \rightarrow p_{2}\|\nabla u\|_{L^{2}}^{2}$.

Set $m=p_{2}\|\nabla u\|_{L^{2}}^{2}$ and $M=\|\nabla u\|_{L^{2}}^{2}$. Then we deduce from Lemma 4.1 that there exist $V \in H^{1}$ and $\left\{x_{n}\right\}_{n=1}^{\infty} \subset \mathbb{R}^{N}$ such that, up to a subsequence,

$$
v_{n}\left(\cdot+x_{n}\right)=\rho_{n}^{N / 2} \psi\left(t_{n}, \rho_{n}\left(\cdot+x_{n}\right)\right) \rightarrow V \quad \text { weakly in } H^{1}
$$

with

$$
\|V\|_{L^{2}} \geq\|u\|_{L^{2}} .
$$


Therefore, we have

$$
\begin{aligned}
\liminf _{n \rightarrow \infty} \int_{|x| \leq r}\left|v_{n}\left(t_{n}, x+x_{n}\right)\right|^{2} d x & =\liminf _{n \rightarrow \infty} \int_{|x| \leq r} \rho_{n}^{N}\left|\psi\left(t_{n}, \rho_{n}\left(x+x_{n}\right)\right)\right|^{2} d x \\
& \geq \int_{|x| \leq r}|V(x)|^{2} d x, \quad \text { for every } r>0 .
\end{aligned}
$$

From the assumption on $a(t)$, we have

$$
\frac{a\left(t_{n}\right)}{\rho_{n}}=\frac{a\left(t_{n}\right)\left\|\nabla \psi\left(t_{n}\right)\right\|_{L^{2}}}{\|\nabla u\|_{L^{2}}} \rightarrow \infty, \quad \text { as } n \rightarrow \infty .
$$

Then $r \rho_{n}<a\left(t_{n}\right)$ for sufficiently large $n$. Therefore, it follows from (4.5) that

$$
\begin{aligned}
& \liminf _{n \rightarrow \infty} \sup _{y \in \mathbb{R}^{N}} \int_{|x-y| \leq a\left(t_{n}\right)}\left|\psi\left(t_{n}, x\right)\right|^{2} d x \\
& \quad \geq \liminf _{n \rightarrow \infty} \sup _{y \in \mathbb{R}^{N}} \int_{|x-y| \leq r \rho_{n}}\left|\psi\left(t_{n}, x\right)\right|^{2} d x \\
& \quad \geq \liminf _{n \rightarrow \infty} \int_{\left|x-x_{n}\right| \leq r \rho_{n}}\left|\psi\left(t_{n}, x\right)\right|^{2} d x \\
& \quad=\liminf _{n \rightarrow \infty} \int_{|x| \leq r} \rho_{n}^{N}\left|\psi\left(t_{n}, \rho_{n}\left(x+x_{n}\right)\right)\right|^{2} d x .
\end{aligned}
$$

This and (4.7) imply that

$$
\liminf _{n \rightarrow \infty} \sup _{y \in \mathbb{R}^{N}} \int_{|x-y| \leq a\left(t_{n}\right)}\left|\psi\left(t_{n}, x\right)\right|^{2} d x \geq \int_{\mathbb{R}^{N}}|V(x)|^{2} d x \geq \int_{\mathbb{R}^{N}}|u(x)|^{2} d x .
$$

Since the sequence $\left\{t_{n}\right\}_{n=1}^{\infty}$ is arbitrary, it follows that

$$
\liminf _{t \rightarrow T^{*}} \sup _{y \in \mathbb{R}^{N}} \int_{|x-y| \leq a(t)}|\psi(t, x)|^{2} d x \geq \int_{\mathbb{R}^{N}}|u(x)|^{2} d x .
$$

Furthermore, for every $t \in\left[0, T^{*}\right)$, the function $y \mapsto h(y)=\int_{|x-y| \leq a(t)}|\psi(t, x)|^{2} d x$ is continuous and $h(y) \rightarrow 0$ as $|y| \rightarrow \infty$. Hence, there is $x(t) \in \mathbb{R}^{N}$ such that

$$
\sup _{y \in \mathbb{R}^{N}} \int_{|x-y| \leq a(t)}|\psi(t, x)|^{2} d x=\int_{|x-x(t)| \leq a(t)}|\psi(t, x)|^{2} d x,
$$

which, together with (4.8), implies (4.1).

In the following, we will study some properties of blow-up solutions to (1.1) with $\left\|\psi_{0}\right\|_{L^{2}}=\|u\|_{L^{2}}$. When $p=2$ or $\alpha=2$, the uniqueness of the ground state of (1.4) plays an important role in the characterization of blow-up solutions to $(1.2)$ in $[7,10]$. However, the uniqueness of ground states of (1.4) with $0<\alpha<N$ and $1+\frac{\alpha}{N}<p_{2}<\frac{N+\alpha}{N-2}$ is not known, we cannot apply the method in $[7,10]$ to study the dynamics of the blow-up solutions.

Theorem 4.3 Assume that $\psi_{0} \in \Sigma, \lambda_{1}=1, \lambda_{2}=-1,0<p_{1}<\frac{4}{N}$ and $p_{2}=1+\frac{2+\alpha}{N}$. Let the solution $\psi(t)$ to (1.1) blow up at the finite time $T^{*}$ and $\left\|\psi_{0}\right\|_{L^{2}}=\|u\|_{L^{2}}$. Then there exists 
$x_{0} \in \mathbb{R}^{N}$ such that

$$
|\psi(t, x)|^{2} \rightarrow\|u\|_{L^{2}}^{2} \delta_{x_{0}} \quad \text { as } t \rightarrow T^{*}
$$

in the sense of a distribution.

Proof Firstly, it follows from Theorem 4.2 that for all $r>0$

$$
\liminf _{t \rightarrow T^{*}} \int_{|x-x(t)|<r}|\psi(t, x)|^{2} d x \geq\|u\|_{L^{2}}^{2} .
$$

This and (2.1) yield for all $r>0$

$$
\|u\|_{L^{2}}^{2}=\left\|\psi_{0}\right\|_{L^{2}}^{2}=\|\psi(t)\|_{L^{2}}^{2} \geq \liminf _{t \rightarrow T^{*}} \int_{|x-x(t)|<r}|\psi(t, x)|^{2} d x \geq\|u\|_{L^{2}}^{2} .
$$

This implies

$$
|\psi(t, x+x(t))|^{2} \rightarrow\|u\|_{L^{2}}^{2} \delta_{x=0} \quad \text { as } t \rightarrow T^{*} .
$$

On the other hand, it follows from the inequality (2.6) and (4.3) that for any $\varepsilon>0$ and any real-valued function $\theta$

$$
\begin{aligned}
H\left(e^{ \pm i \epsilon \theta} \psi(t)\right)= & \frac{\epsilon^{2}}{2} \int_{\mathbb{R}^{N}}|\psi(t, x)|^{2}|\nabla \theta(x)|^{2} d x \\
& \mp \epsilon \operatorname{Im} \int_{\mathbb{R}^{N}} \bar{\psi}(t, x) \nabla \psi(t, x) \cdot \nabla \theta(x) d x+H(\psi(t)) \\
\geq & \frac{1}{2} \int_{\mathbb{R}^{N}}\left|\nabla\left(e^{ \pm i \epsilon \theta} \psi(t, x)\right)\right|^{2} d x\left(1-\frac{\left\|\psi_{0}\right\|_{L^{2}}^{2 p_{2}-2}}{\|u\|_{L^{2}}^{2 p_{2}-2}}\right)=0 .
\end{aligned}
$$

This implies that

$$
\begin{aligned}
\mid \mp & \operatorname{Im} \int_{\mathbb{R}^{N}} \bar{\psi}(t, x) \nabla \psi(t, x) \cdot \nabla \theta(x) d x \mid \\
& \leq\left(2 H(\psi(t)) \int_{\mathbb{R}^{N}}|\psi(t, x)|^{2}|\nabla \theta(x)|^{2} d x\right)^{1 / 2} .
\end{aligned}
$$

Therefore, this and $H(\psi(t)) \leq E(\psi(t))=E\left(\psi_{0}\right)$ yield

$$
\begin{aligned}
\left.\left|\frac{d}{d t} \int_{\mathbb{R}^{N}}\right| \psi(t, x)\right|^{2} x_{j} d x \mid & \leq C\left|\int_{\mathbb{R}^{N}} \bar{\psi}(t, x) \partial_{j} \psi(t, x) d x\right| \\
& \leq C\left|\int_{\mathbb{R}^{N}} \bar{\psi}(t, x) \nabla \psi(t, x) \nabla x_{j} d x\right| \\
& \leq C\left(2 H(\psi(t)) \int_{\mathbb{R}^{N}}|\psi(t, x)|^{2}\left|\nabla x_{j}\right|^{2} d x\right)^{1 / 2} \leq C,
\end{aligned}
$$

for every $j=1,2, \ldots, N$. This implies

$$
\left.\left|\int_{\mathbb{R}^{N}}\right| \psi\left(t_{m}, x\right)\right|^{2} x_{j} d x-\int_{\mathbb{R}^{N}}\left|\psi\left(t_{k}, x\right)\right|^{2} x_{j} d x|\leq C| t_{m}-t_{k} \mid \rightarrow 0 \quad \text { as } m, k \rightarrow \infty,
$$


for every $j=1,2, \ldots, N$, where $\left\{t_{m}\right\}_{m=1}^{\infty},\left\{t_{k}\right\}_{k=1}^{\infty} \subseteq\left(0, T^{*}\right)$ and $\lim _{m \rightarrow \infty} t_{m}=\lim _{k \rightarrow \infty} t_{k}=T^{*}$. Thus, we have

$$
\lim _{t \rightarrow T^{*}} \int_{\mathbb{R}^{N}}|\psi(t, x)|^{2} x_{j} d x \quad \text { exists, }
$$

for every $j=1,2, \ldots, N$. Set

$$
x_{0}=\lim _{t \rightarrow T^{*}} \int_{\mathbb{R}^{N}}|\psi(t, x)|^{2} x d x /\|u\|_{L^{2}}^{2},
$$

it follows that

$$
\lim _{t \rightarrow T^{*}} \int_{\mathbb{R}^{N}}|\psi(t, x)|^{2} x d x=\|u\|_{L^{2}}^{2} x_{0}
$$

In addition, we deduce from Lemma 2.2 and (4.11) that

$$
\begin{aligned}
& \int_{\mathbb{R}^{N}}|x|^{2}|\psi(t, x+x(t))|^{2} d x \\
& \quad \leq C \int_{\mathbb{R}^{N}}|x+x(t)|^{2}|\psi(t, x+x(t))|^{2} d x+C|x(t)|^{2} \int_{\mathbb{R}^{N}}|\psi(t, x+x(t))|^{2} d x \\
& \quad \leq C+C|x(t)|^{2}\left\|\psi_{0}\right\|_{L^{2}}^{2} \\
& \quad \leq C+C \limsup _{t \rightarrow T^{*}} \int_{|x|<1}|x+x(t)|^{2}|\psi(t, x+x(t))|^{2} d x \\
& \quad \leq C+C \int_{\mathbb{R}^{N}}|x|^{2}|\psi(t, x)|^{2} d x \leq C .
\end{aligned}
$$

This implies

$$
\limsup _{t \rightarrow T^{*}}|x(t)| \leq \frac{\sqrt{C}}{\left\|\psi_{0}\right\|_{L^{2}}}
$$

and

$$
\limsup _{t \rightarrow T^{*}} \int_{\mathbb{R}^{N}}|x|^{2}|\psi(t, x+x(t))|^{2} d x \leq C .
$$

Thus, for any $\varepsilon>0$, there is $R_{0}$ such that

$$
\left.\limsup _{t \rightarrow T^{*}}\left|\int_{|x| \geq R_{0}} x\right| \psi(t, x+x(t))\right|^{2} d x \mid \leq \frac{C}{R_{0}}<\frac{\varepsilon}{2} .
$$

We see from (4.11) that

$$
\begin{aligned}
& \left.\underset{t \rightarrow T^{*}}{\limsup }\left|\int_{\mathbb{R}^{N}}\right| \psi(t, x)\right|^{2} x d x-x(t)\|u\|_{L^{2}}^{2} \mid \\
& \quad=\left.\limsup _{t \rightarrow T^{*}}\left|\int_{\mathbb{R}^{N}}\right| \psi(t, x)\right|^{2}(x-x(t)) d x \mid \\
& \quad \leq\left.\limsup _{t \rightarrow T^{*}}\left|\int_{|x| \leq R_{0}}\right| \psi(t, x+x(t))\right|^{2} x d x \mid+\frac{\varepsilon}{2} \leq \varepsilon .
\end{aligned}
$$


This and (4.14) imply that $\lim _{t \rightarrow T^{*}} x(t)=x_{0}$. Thus, it follows from (4.11) that

$$
|\psi(t, x)|^{2} \rightarrow\|u\|_{L^{2}}^{2} \delta_{x=x_{0}} \quad \text { as } t \rightarrow T^{*}
$$

in the sense of distribution.

Finally, we study the blow-up rate of blow-up solutions to (1.1) with $\left\|\psi_{0}\right\|_{L^{2}}=\|u\|_{L^{2}}$.

Theorem 4.4 Assume that $\psi_{0} \in \Sigma, \lambda_{1}=1, \lambda_{2}=-1,0<p_{1}<\frac{4}{N}$ and $p_{2}=1+\frac{2+\alpha}{N}$. Let the solution $\psi(t)$ to (1.1) blow up at the finite time $T^{*}$ and $\left\|\psi_{0}\right\|_{L^{2}}=\|u\|_{L^{2}}$. Then there exists a constant $C>0$ such that for all $t \in\left[0, T^{*}\right)$

$$
\|\nabla \psi(t)\|_{L^{2}} \geq \frac{C}{T^{*}-t}
$$

Proof Let $g \in C_{0}^{\infty}\left(\mathbb{R}^{N}\right)$ be a nonnegative radial function satisfying

$$
g(x)=g(|x|)=|x|^{2}, \quad \text { if }|x|<1 \quad \text { and } \quad|\nabla g(x)|^{2} \leq C g(x) .
$$

For $A>0$, we define $g_{A}(x)=A^{2} g\left(\frac{x}{A}\right)$ and $h_{A}(t)=\int_{\mathbb{R}^{N}} g_{A}\left(x-x_{0}\right)|\psi(t, x)|^{2} d x$ with $x_{0}$ defined by (4.13).

It follows from (4.12) and $H(\psi(t)) \leq E(\psi(t))=E\left(\psi_{0}\right)$ that for every $t \in\left[0, T^{*}\right)$

$$
\begin{aligned}
\left|\frac{d}{d t} h_{A}(t)\right| & \leq C\left|\int_{\mathbb{R}^{N}} \bar{\psi}(t, x) \nabla \psi(t, x) \nabla g_{A}\left(x-x_{0}\right) d x\right| \\
& \leq 2 \sqrt{H(\psi(t))}\left(\int_{\mathbb{R}^{N}}|\psi(t, x)|^{2}\left|\nabla g_{A}\left(x-x_{0}\right)\right|^{2} d x\right)^{1 / 2} \\
& \leq 2 \sqrt{E\left(\psi_{0}\right)}\left(\int_{\mathbb{R}^{N}}|\psi(t, x)|^{2}\left|g_{A}\left(x-x_{0}\right)\right| d x\right)^{1 / 2} \\
& \leq C \sqrt{h_{A}(t)} .
\end{aligned}
$$

This implies that there is a constant $C$ such that $\left|\frac{d}{d t} \sqrt{h_{A}(t)}\right| \leq C$. Integrating on both sides with respect to time $t$ on $\left[t_{1}, t\right]$, we have

$$
\left|\sqrt{h_{A}(t)}-\sqrt{h_{A}\left(t_{1}\right)}\right| \leq C\left|t-t_{1}\right| \text {. }
$$

On the other hand, from (4.9), we have

$$
h_{A}\left(t_{1}\right) \rightarrow\|Q\|_{L^{2}} g_{A}(0)=0 \quad \text { as } t_{1} \rightarrow T^{*} .
$$

Thus, let $t_{1} \rightarrow T^{*}$ in (4.20), we have $h_{A}(t) \leq C\left(T^{*}-t\right)^{2}$. Now fix $t \in\left[0, T^{*}\right)$, it follows that

$$
\lim _{A \rightarrow \infty} h_{A}(t)=\int_{\mathbb{R}^{N}}\left|x-x_{0}\right|^{2}|\psi(t, x)|^{2} d x \leq C\left(T^{*}-t\right)^{2} .
$$

Thus, we deduce from the uncertainty principle that

$$
\|\nabla \psi(t)\|_{L^{2}} \geq \frac{\int_{\mathbb{R}^{N}}|\psi(x)|^{2} d x}{\left(\int_{\mathbb{R}^{N}}\left|x-x_{0}\right|^{2}|\psi(x)|^{2} d x\right)^{1 / 2}} \geq \frac{C}{T^{*}-t}, \quad \forall t \in\left[0, T^{*}\right) .
$$

This completes the proof. 


\section{Conclusions}

In this paper, we study the dynamics of blow-up solutions for the nonlinear SchrödingerChoquard equation (1.1) with $0<p_{1}<\frac{4}{N}$ and $p_{2}=1+\frac{2+\alpha}{N}$. In the previous papers, the scale invariance played an important role in the study of the dynamics of blow-up solutions to nonlinear Schrödinger equations. Because there exists no scale invariance for Eq. (1.1), the study of blow-up solutions to (1.1) is an interesting problem. We must overcome the difficulty brought about by the loss of scale invariance. For (1.1), we find that the ground state solution $u$ to (1.4) exactly describes the sharp threshold mass of global existence and blow-up, the dynamical properties of blow-up solutions, including $L^{2}$-concentration, limiting profile and blow-up rates.

\section{Acknowledgements}

This work is supported by the PhD scientific research start-up capital funded projects of Longdong University (XYBY05), the fundamental research funds for the Gansu universities (2015A-150).

\section{Funding}

Not applicable.

\section{Abbreviations}

Not applicable.

\section{Availability of data and materials}

Not applicable.

\section{Competing interests}

The authors declare that no competing interests exist.

Authors' contributions

The authors contributed equally to this paper. All authors read and approved the final manuscript.

\section{Publisher's Note}

Springer Nature remains neutral with regard to jurisdictional claims in published maps and institutional affiliations.

Received: 28 November 2017 Accepted: 19 April 2018 Published online: 27 April 2018

References

1. Cazenave, T: Semilinear Schrödinger Equations. Courant Lecture Notes in Mathematics, vol. 10. AMS, Providence (2003)

2. Sulem, C., Sulem, P.L.: The Nonlinear Schrödinger Equation. Springer, Berlin (1999)

3. Chen, J., Guo, B.: Strong instability of standing waves for a nonlocal Schrödinger equation. Phys. D: Nonlinear Phenom. 227, 142-148 (2007)

4. Feng, B., Zhao, D., Sun, C.: On the Cauchy problem for the nonlinear Schrödinger equations with time-dependent linear loss/gain. J. Math. Anal. Appl. 416, 901-923 (2014)

5. Feng, B., Zhang, H.: Stability of standing waves for the fractional Schrödinger-Choquard equation. Comput. Math. Appl. 75, 2499-2507 (2018)

6. Feng, B., Zhang, H.: Stability of standing waves for the fractional Schrödinger-Hartree equation. J. Math. Anal. Appl. 460, 352-364 (2018)

7. Genev, H., Venkov, G.: Soliton and blow-up solutions to the time-dependent Schrödinger-Hartree equation. Discrete Contin. Dyn. Syst., Ser. S 5, 903-923 (2012)

8. Liu, J., Qian, A.: Ground state solution for a Schrödinger-Poisson equation with critical growth. Nonlinear Anal., Real World Appl. 40, 428-443 (2018)

9. Mao, A., Yang, L., Qian, A., Luan, S.: Existence and concentration of solutions of Schrödinger-Poisson system. Appl. Math. Lett. 68, 8-12 (2017)

10. Miao, C., Xu, G., Zhao, L.: On the blow up phenomenon for the mass critical focusing Hartree equation in $\mathbb{R}^{4}$. Colloq Math. 119, 23-50 (2010)

11. d'Avenia, P., Squassina, M.: Soliton dynamics for the Schrödinger-Newton system. Math. Models Methods Appl. Sci. 24, 553-572 (2014)

12. Guo, Q., Zhu, S.: Sharp threshold of blow-up and scattering for the fractional Hartree equation. J. Differ. Equ. 264, 2802-2832 (2018)

13. Zhu, S.: On the Davey-Stewartson system with competing nonlinearities. J. Math. Phys. 57, 031501 (2016)

14. Zhu, S.: On the blow-up solutions for the nonlinear fractional Schrödinger equation. J. Differ. Equ. 261, 1506-1531 (2016)

15. Zhang, J., Zhu, S.: Stability of standing waves for the nonlinear fractional Schrödinger equation. J. Dyn. Differ. Equ. 29, 1017-1030 (2017) 
16. Zhu, S.: Existence of stable standing waves for the fractional Schrödinger equations with combined nonlinearities. J. Evol. Equ. 17, 1003-1021 (2017)

17. Bonanno, C., d'Avenia, P., Ghimenti, M., Squassina, M.: Soliton dynamics for the generalized Choquard equation. J. Math. Anal. Appl. 417, 180-199 (2014)

18. Feng, B., Yuan, X.: On the Cauchy problem for the Schrödinger-Hartree equation. Evol. Equ. Control Theory 4, 431-445 (2015)

19. Feng, B.: Sharp threshold of global existence and instability of standing wave for the Schrödinger-Hartree equation with a harmonic potential. Nonlinear Anal., Real World Appl. 31, 132-145 (2016)

20. Merle, F., Raphaël, P.: Blow-up dynamic and upper bound on the blow-up rate for critical nonlinear Schrödinger equation. Ann. Math. 16, 157-222 (2005)

21. Zhu, S., Zhang, J., Yang, H.: Limiting profile of the blow-up solutions for the fourth-order nonlinear Schrödinger equation. Dyn. Partial Differ. Equ. 7, 187-205 (2010)

22. Moroz, V., Schaftingen, J.: Groundstates of nonlinear Choquard equations: existence, qualitative properties and decay asymptotics. J. Funct. Anal. 265, 153-184 (2013)

23. Li, X., Zhang, J., Lai, S., Wu, Y.: The sharp threshold and limiting profile of blow-up solutions for a Davey-Stewartson system. J. Differ. Equ. 250, 2197-2226 (2011)

24. Weinstein, M.I.: Nonlinear Schrödinger equations and sharp interpolation estimates. Commun. Math. Phys. 87 567-576 (1983)

25. Feng, B:: On the blow-up solutions for the nonlinear Schrödinger equation with combined power-type nonlinearities J. Evol. Equ. 18, 203-220 (2018)

\section{Submit your manuscript to a SpringerOpen ${ }^{\circ}$ journal and benefit from:}

- Convenient online submission

- Rigorous peer review

- Open access: articles freely available online

- High visibility within the field

- Retaining the copyright to your article

Submit your next manuscript at $\gg$ springeropen.com 